\title{
Predictive value of coagulation factor XIII on bleeding risk in ischemic stroke patients treated with intravenous thrombolysis
}

\author{
Linjing Zhang ${ }^{1 *}$, Chi Zhang ${ }^{2 \#}$, Yi Luo $^{3 \wedge}$, Ning Tang ${ }^{4}$ \\ ${ }^{1}$ Department of Clinical Laboratory, Huangzhou District People's Hospital, Huanggang, China; ${ }^{2}$ Department of Clinical Laboratory, Tongji \\ Hospital, Tongji Medical College, Huazhong University of Science and Technology, Wuhan, China; ${ }^{3}$ Department of Laboratory Medicine, \\ Zhongnan Hospital of Wuhan University, Wuhan, China; ${ }^{4}$ Department of Clinical Laboratory, Tongji Hospital, Tongji Medical College, Huazhong \\ University of Science and Technology, Wuhan, China \\ Contributions: (I) Conception and design: N Tang; (II) Administrative support: None; (III) Provision of study materials or patients: None; (IV) \\ Collection and assembly of data: L Zhang, C Zhang, Y Luo; (V) Data analysis and interpretation: L Zhang, C Zhang, Y Luo; (VI) Manuscript \\ writing: All authors; (VII) Final approval of manuscript: All authors. \\ \#These authors contributed equally to this work. \\ Correspondence to: Dr. Ning Tang. Tongji Hospital, Tongji Medical College, Huazhong University of Science and Technology, 1095 Jiefang Ave, \\ Wuhan 430030, China. Email: tonyjesus@126.com; Dr. Yi Luo. Department of Laboratory Medicine, Zhongnan Hospital of Wuhan University, 169 \\ Donghu Road, Wuhan 430071, China. Email: luoyi929@aliyun.com.
}

Background: Intravenous thrombolysis (IVT) therapy with recombinant tissue plasminogen activator (rtPA) in acute ischemic stroke (AIS) has a known risk of intracerebral hemorrhage (ICH). We aimed to identify the predictive value of coagulation factor XIII (FXIII) on post-thrombolytic ICH risk in AIS patients.

Methods: The study cohort included 69 diagnosed AIS patients undergoing IVT treatment within 24 hours of symptom onset. Blood samples taken on admission were analyzed for FXIII antigen levels with an automated latex enhanced immunoassay. Conventional coagulation parameters including prothrombin time (PT), activated partial thromboplastin time (APTT), fibrinogen (FIB), and D-dimer (DD) were also tested.

Results: Of the 69 AIS patients, 23 (33.3\%) developed post-thrombolytic ICH. Plasma FXIII levels showed a significant decrease, accompanied with elevated FIB and DD levels in AIS patients with post-thrombolytic ICH. Multivariable logistic regression (LR) revealed that FXIII and FIB were independently associated with post-thrombolytic ICH progression. Area under receiver operating characteristic curve of FXIII selected by the further forward logistic regression was 0.823 [95\% confidence interval (CI): 0.712-0.904], and the cutoff value of $76.6 \%$ yielded good sensitivity at $91.3 \%$ and good negative predictive value (NPV) at $93.9 \%$.

Conclusions: Our findings indicated that plasma FXIII level may be an independent determinant for predicting post-thrombolytic bleeding risk in AIS patients.

Keywords: Acute ischemic stroke (AIS); coagulation factor XIII (FXIII); intravenous thrombolysis (IVT); postthrombolytic intracerebral hemorrhage (post-thrombolytic ICH); predictive marker

Submitted Apr 12, 2021. Accepted for publication Jun 23, 2021.

doi: 10.21037/apm-21-1174

View this article at: https://dx.doi.org/10.21037/apm-21-1174

$\wedge$ ORCID: 0000-0001-6304-5626. 


\section{Introduction}

Acute ischemic stroke (AIS) is a leading cause of death and disability worldwide (1). Currently, the most effective and clinically recognized therapy to improve functional outcomes is early thrombolysis guided by a time window using recombinant tissue plasminogen activator (rtPA), alteplase, urokinase, and so on $(2,3)$. Before receiving intravenous thrombolysis (IVT), AIS patients are carefully screened to minimize bleeding risk according to the contraindications set forth in clinical guidelines (4). However, post-thrombolytic intracerebral hemorrhage (ICH) is still inevitable and fatal in approximately 7-8\% of AIS patients (4-6). Many clinical and radiological variables, along with clinical laboratory indicators, have been reported to predict post-thrombolytic ICH (7-9). Among these, several biomarkers reflecting the states of coagulation and functions of fibrinolysis may be associated with hemorrhagic transformation (10-12).

Blooding coagulation factor XIII (FXIII), also called fibrin-stabilizing factor, is a tetrameric protein consisting of 2 potentially active A-subunits and $2 \mathrm{~B}$-subunits (carrier proteins) that circulate in plasma $(13,14)$. FXIII is essential for maintaining hemostasis due to its role in fibrin stabilization and in the protection of fibrin from proteolytic degradation by the fibrinolytic system $(15,16)$. Thus, its activity is associated with A-subunits and fibrinogen concentrations (17). Growing evidence has shown that the role of FXIII as a risk factor in thrombotic disorders; however, reports on the relation of ischemic stroke and FXIII are still limited (16). Previous studies have shown that a polymorphism in the A-subunit gene (FXIII Val34Leu) is protective against occlusive vascular diseases, and low concentrations of FXIII A-subunit may predict poor outcome in stroke subjects $(18,19)$. Recently, it has been reported that low levels of FXIII after IVT can predict short-term mortality in AIS patients (5), whereas the predictive value of FXIII levels before IVT on the risk of ICH is still vague. Herein, we aimed to evaluate the impact of blood FXIII levels measured before the course of thrombolysis in AIS patients on the occurrence of postthrombolytic ICH. We present the following article in accordance with the STROBE reporting checklist (available at https://dx.doi.org/10.21037/apm-21-1174).

\section{Methods}

\section{Study population}

This study was retrospective in design and was conducted between October 2019 and April 2020. A total of 69 AIS patients undergoing IVT were recruited in the Department of Neurology of Tongji Hospital of Huazhong University of Science and Technology. AIS was defined as a sudden onset of nonconvulsive and focal neurological deficit. As described in our previous clinical study (20), the inclusion criteria of AIS patients were clinical presentation of first-ever stroke, age 18 years or older, and a diagnosis via brain imaging of acute cerebral infarction. Meanwhile, the exclusion criteria were the following: cerebral hemorrhage, subdural hematoma, intracranial space-occupying lesions, traumatic cerebrovascular damage, heart failure, malignant diseases, immunologic diseases, severe infection, or pregnancy. Most enrolled patients in this study suffered a large vessel thrombosis, according to the classification criteria of the Trial of Org 10172 in Acute Stroke Treatment (TOAST) (21). The average time from symptom onset to treatment with recombinant tissue plasminogen activator (rtPA) was less than 24 hours, and the duration of thrombolysis was approximately 1 hour for each patient. According to ICH occurrence after rtPA treatment, all enrolled patients were further divided into non-bleeding and bleeding AIS subgroups. All procedures performed in this study involving human participants were in accordance with the Declaration of Helsinki (as revised in 2013). The study was approved by Medicine Research Ethics Committee of Tongji Hospital of Huazhong University of Science and Technology (No. TJ-IRB20192511), and all stroke patients gave informed consent before enrollment.

\section{Clinical assessment}

The medical information of all patients was collected, including age, sex, risk factors, common comorbidities, standard laboratory tests, and previous medical history. Physical and neurological examinations, and cerebral computed tomography (CT) or magnetic resonance imaging (MRI) were also performed. Neurological severity was assessed on admission according to the National Institutes of Health Stroke Scale (NIHSS) score and Glasgow Coma Scale (GCS) score $(22,23)$. Risk factors like hypertension, diabetes mellitus, and related heart diseases were determined by the corresponding diagnostic criteria combined with medical history. Occurrence of postthrombolytic ICH was routinely assessed by cerebral CT or MRI, after which the AIS patients were divided into the bleeding group and non-bleeding group. 


\section{Sample collection and laboratory test}

Blood samples of sodium citrate anticoagulant were collected from each of the 69 AIS patients within 24 hours before thrombolytic therapy. Following this, the plateletpoor plasma was separated, and the coagulation factors in plasma samples were tested immediately within 2 hours. Quantitative determination of FXIII antigen in human citrated plasma was tested by latex enhanced immunoassay on an ACL TOP automated coagulation analyzer using a commercially available reagent kit (Factor XIII Antigen, Hemosil, Instrumentation Laboratory Company, Bedford, MA, USA; reference range 75.2-154.8\%, CV $3.5 \%)$. Conventional coagulation parameters including prothrombin time (PT), activated partial thromboplastin time (APTT), fibrinogen (FIB), and D-dimer (DD) were also detected by routine methods from the same sample.

\section{Statistical analysis}

SPSS 19.0 (IBM Corp., Armonk, NY, USA) and GraphPad Prism 7.0 (GraphPad Software, San Diego, CA, USA) were used for statistical analysis. Descriptive data are presented as mean \pm standard deviation $(\mathrm{SD})$, median (interquartile range, IQR), or proportions. Between-group and across-group differences were compared by Student's $t$-test, Mann-Whitney $\mathrm{U}$ test, one-way analysis of variance (ANOVA), and Kruskal-Wallis test or $\chi^{2}$ test, where appropriate. Multivariable logistic regression (LR) using the Wald test was performed to study which variables were independently associated with ICH developed after IVT in stroke. Additionally, forward LR was employed to select determinants of ICH to build a predictive model. A 2-sided $\mathrm{P}$ value $<0.05$ was considered statistically significant.

\section{Results}

\section{Clinical characteristics of enrolled AIS patients with and without post-thrombolytic bleeding}

The clinical background of the AIS patients with and without post-thrombolytic ICH is shown in Table 1. After IVT with rtPA, ICH developed in 23 of 69 (33.3\%) AIS patients. The gender and age distribution did not differ significantly between the bleeding and non-bleeding AIS group. No obvious difference was found in the incident of risk factors, such as hypertension, diabetes, related heart diseases, and other common complications. Compared to the AIS patients without post-thrombolytic bleeding, AIS patients with post-thrombolytic bleeding had significantly higher NIHSS and GCS scores, suggesting more severe neurological function deficits.

\section{AIS patients with post-thrombolytic ICH had lower FXIII levels detected before thrombolysis}

We first analyzed the baseline changes of coagulation parameters before thrombolysis, including PT, APTT, FIB, DD, and FXIII. The results found that the FXIII antigen levels on admission (before thrombolysis) showed a significant decrease (59.9\%; IQR $55.0-78.5 \%$ ) in AIS patients with post-thrombolytic ICH, compared with the non-bleeding AIS patients (85.5\%; IQR 73.8-94.5\%). Conversely, the levels of plasma FIB and DD in the patients with post-thrombolytic ICH were obviously higher than those in the non-bleeding AIS patients (Table 1).

\section{Plasma FXIII antigen level may be an independent determinant for predicting post-tbrombolytic bleeding in AIS patients}

Multivariable LR using the Wald test was performed to calculate the likelihood ratio (LR) of the variables that changed between the non-bleeding and bleeding AIS groups. Considering that (I) age in stroke patients has been associated with a higher risk of development of ICH after IVT $(24,25)$, that (II) male sex has been reported to be independently associated with ICH (26), and (III) biological plausibility, we also included age and sex in the multivariable model. The results showed that the FIB and FXIII levels were significantly associated with post-thrombolytic ICH progression, independent of other coagulation and biological covariates (Table 2).

The further forward LR method suggested that the FXIII level $(\mathrm{P}<0.001)$ but not FIB $(\mathrm{P}=0.062)$ was an independent determinant for predicting ICH after thrombolysis. The receiver operating characteristic (ROC) curve showed that the area under curve (AUC) of FXIII on predicting bleeding risk of AIS patients treated with IVT was 0.823 (Figure 1); furthermore, the optimal cutoff value of $76.6 \%$ yielded good sensitivity at $91.3 \%$, with specificity at $67.4 \%$, positive predictive value (PPV) at $58.3 \%$, and negative predictive value (NPV) at $93.9 \%$ (Table 3).

\section{Discussion}

The present study aimed to determine whether a lower 
Table 1 Baseline characteristics of the study population $(\mathrm{n}=69)$

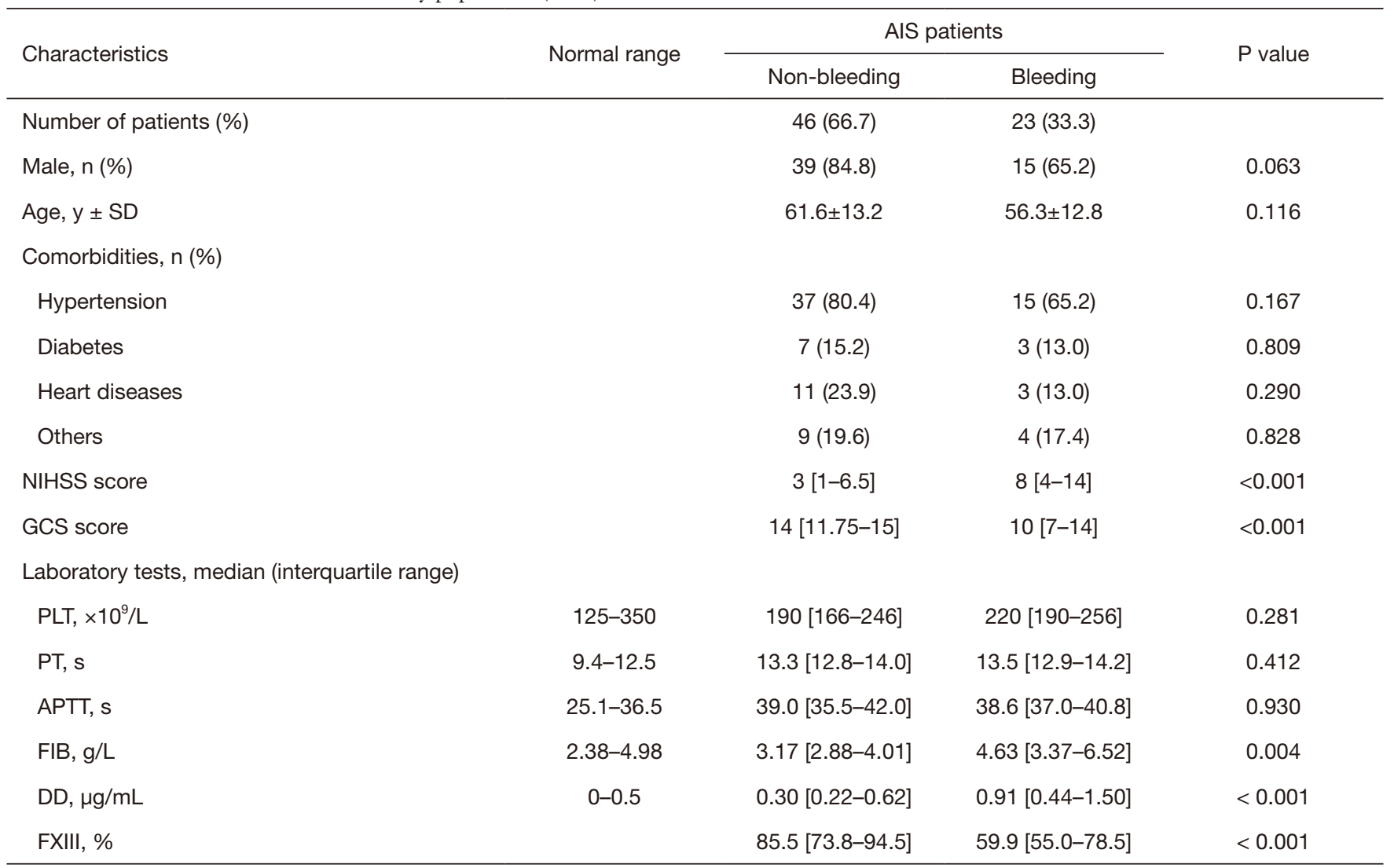

Data reported as number (\%), mean \pm standard deviation (SD) or median [interquartile range, IQR]. P<0.05, considered statistically significant. AIS, acute ischemic stroke; NIHSS, National Institutes of Health Stroke Scale; GCS, Glasgow Coma Scale; PLT, platelets; PT, prothrombin time; APTT, activated partial thromboplastin time; FIB, fibrinogen; DD, D-dimer; FXIII, coagulation factor XIII.

plasma FXIII level in AIS patients before thrombolysis is an independent determinant for predicting post-thrombolytic ICH. To our knowledge, this may be the first study on clinical determinants of predicting ICH progression in stroke patients treated with IVT. Considering the high overall mortality and morbidity of bleeding in AIS patients, it is essential at an early stage to identify patients at a higher risk of developing ICH after IVT-rtPA. Several prognostic scores have been used to identify AIS patients with a high risk of post-thrombolysis ICH, such as NIHSS and GCS score $(27,28)$. Nevertheless, the value of these scores for individualized outcome predictions is limited due to the dichotomization or categorization of continuous variables, and results have varied across different studies $(29,30)$. The recent multiple prognostic scoring systems were investigated and mainly used to predict the clinical outcome, such as SPAN-100 index, the GPASPS score, in which no coagulation markers were included $(27,28,31)$. Thus, the possible coagulation defects cannot be reflected when using these systems. Maybe the effective coagulation indicators combinated with scoring system can improve the accuracy of predicting hemorrage risk. Among conventional coagulation markers, high D-dimer concentrations have been reported to be associated with both cardiovascular events and major bleeding during anticoagulant therapy (32). Thus, the cardiovascular risk factors must be taken into consideration when investigating the relationship between D-dimer and ICH (33). Previously, a high level of fibrinogen degradation factors (FDPs) and decreased fibrinogen at 2 hours after thrombolysis was found, called "early fibrinogen degradation coagulopathy", which was predictive of early ICH (34). However, potential predictive factors for assessing the bleeding risk before thrombolysis in the earlier stage of AIS patients have not been definitively clarified.

It has been suggested that one of the mechanisms 
Table 2 Coagulation and biological variables associated with postthrombolytic ICH in multivariable analysis

\begin{tabular}{lcc}
\hline Variable & OR $(95 \% \mathrm{Cl})$ & $\mathrm{P}$ value \\
\hline Age at AIS diagnosis (years) & $0.957(0.895-1.023)$ & 0.196 \\
Male sex & $4.299(0.767-24.095)$ & 0.097 \\
GCS score & $1.041(0.634-1.710)$ & 0.874 \\
NIHSS score & $1.111(0.829-1.488)$ & 0.482 \\
FIB & $2.177(1.021-4.639)$ & 0.044 \\
DD & $1.434(0.673-3.057)$ & 0.351 \\
FXIII & $0.919(0.870-0.971)$ & 0.003 \\
\hline
\end{tabular}

$\mathrm{P}<0.05$, considered statistically significant. $\mathrm{ICH}$, intracerebral hemorrhage; AIS, acute ischemic stroke; LR, likelihood ratio; $\mathrm{CI}$, confident interval; NIHSS, National Institutes of Health Stroke Scale; GCS, Glasgow Coma Scale; FIB, fibrinogen; DD, D-dimer; FXIII, coagulation factor XIII.

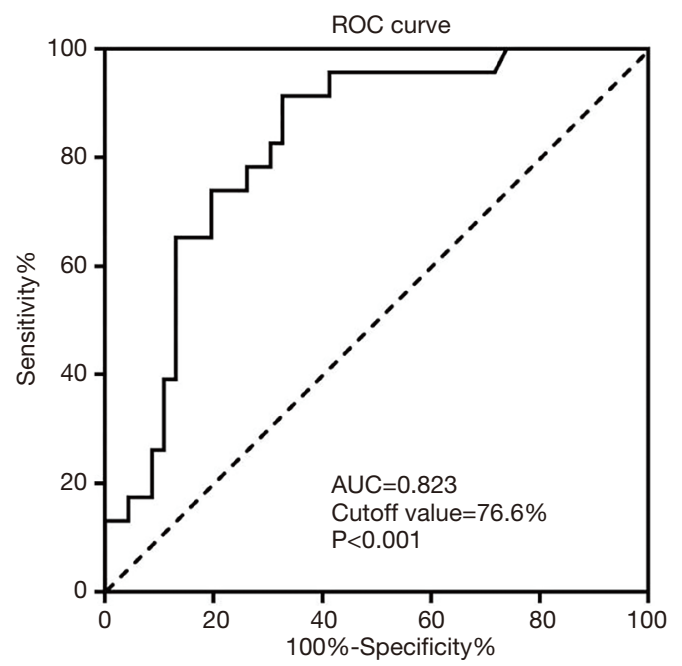

Figure 1 Receiver operating characteristic (ROC) curve in AIS patients with post-thrombolytic ICH. The area under ROC curve was 0.823 for plasma FXIII level. AIS, acute ischemic stroke; ICH, intracerebral hemorrhage; FXIII, coagulation factor XIII.

of ICH may be a defect in anti-fibrinolysis through an excessive consumption of anti-fibrinolytic components (34). One hypothesis speculates that a decrease in the level of fibrinolytic inhibitors, such as the FXIII, or factors participating in clot stability could be useful predictors of ICH (35). However, the levels of FXIII before thrombolysis as evaluated by sandwich enzyme-linked immunosorbent assay (ELISA) have been reported to be within a normal or not-significantly-lower range $(5,35)$. In our study, highly
Table 3 Predictive value of FXIII activity $<76.6 \%$ on bleeding risk in AIS patients treated with IVT

\begin{tabular}{lc}
\hline Variable & Plasma FXIII activity \\
\hline Sensitivity & $91.3 \%$ \\
Specificity & $67.4 \%$ \\
PPV & $58.3 \%$ \\
NPV & $93.9 \%$ \\
AUC & 0.823 \\
$95 \% \mathrm{Cl}$ & $0.712-0.904$ \\
P & $<0.001$ \\
\hline
\end{tabular}

$\mathrm{P}<0.05$, considered statistically significant. FXIII, coagulation factor XIII; AIS, acute ischemic stroke; IVT, intravenous thrombolysis; PPV, positive predictive value; NPV, negative predictive value; AUC, area under curve; $95 \% \mathrm{CI}, 95 \%$ confidence interval.

specific antigen latex enhanced immunoassay was used to capture the A-subunit of FXIII, which indicated a lower FXIII level before thrombolysis present in AIS patients who developed ICH after IVT-rtPA, accompanied by high D-dimer and FIB levels. The mechanism involved in the reduction of FXIII level and activity is still unclear. One hypothesis posits that a considerable amount of FXIII being incorporated into the growing thrombus leads to consumption and/or degradation of FXIII and inactivation by plasmin due to ongoing activity of the coagulation system after stroke (36). One study found that FXIII activity measured before and after thrombolysis was not significantly changed (5), suggesting a potential earlier predictive value. Considering these findings in combination with our data, we speculate that low FXIII antigen activity in AIS patients is associated with high bleeding risk, and should be monitored closely to prevent the development of post-thrombolytic ICH.

The independent association of low FXIII level with a higher risk of post-thrombolytic ICH is novel. According to the multivariable LR, low FXIII and high FIB levels, but not high D-dimer before thrombolytic treatment, were independent factors for predicting $\mathrm{ICH}$, suggesting the high D-dimer may be present at least partly because of concurrent cardiovascular diseases. The NIHSS and GCS scores on admission, commonly used to access the severity of stroke, were higher in the AIS patients with post-thrombolytic ICH. However, these 2 scores were not identified as the independent factors for predicting ICH in 
our study. Data from further forward LR analysis revealed that a low FXIII level may be used as a clinical determinant to predict the ICH risk, whereas a high FIB level before thrombolysis may be not an independent determinant. Moreover, the AUC in ROC curve analysis of FXIII level was 0.823 , which is close to 1 , indicating a superior predictive value in AIS patients with post-thrombolytic ICH. A cutoff value of $76.6 \%$ yielded good sensitivity at $91.3 \%$ and good NPV at $93.9 \%$.

Taken together, plasma FXIII level may be valuable for predicting post-thrombolytic bleeding risk in AIS patients, and low FXIII level should be included in the consideration of thrombolysis contraindications. For AIS patient with low FXIII level, the alternative treatments should be given. Two recent studies have shown a survival increase for FXIII substitution in trauma patients, suggesting the potential effectiveness of FXIII for the treatment of hemorrhage $(37,38)$. Moreover, increased FXIII levels may result in elevated incorporation of $\alpha 2$ plasmin inhibitor ( $\alpha 2-\mathrm{PI})$, showing an association with the outcome of IVT therapy (39). Therapeutic plasma exchange or albumin solutions enriched FXIII, and purified plasma-derived FXIII may restore coagulation potential, which may be a promising treatment strategy and merits further verification $(40,41,42)$. However, some limitations to our study should be addressed. Given the nature of the study design, we were unable to elucidate the mechanism linking low FXIII levels before thrombolysis with post-thrombolytic ICH progression, and additional multicenter studies using larger samples of AIS patients are needed to validate our findings.

\section{Acknowledgments}

Funding: This study was supported by the National Natural Science Foundation of China (No. 82071324).

\section{Footnote}

Reporting Checklist: The authors have completed the STROBE reporting checklist. Available at https://dx.doi. org/10.21037/apm-21-1174

Data Sharing Statement: Available at https://dx.doi. org/10.21037/apm-21-1174

Conflicts of Interest: All authors have completed the ICMJE uniform disclosure form (available at https://dx.doi. org/10.21037/apm-21-1174). The authors have no conflicts of interest to declare.

Ethical Statement: The authors are accountable for all aspects of the work in ensuring that questions related to the accuracy or integrity of any part of the work are appropriately investigated and resolved. All procedures performed in this study involving human participants were in accordance with the Declaration of Helsinki (as revised in 2013). The study was approved by Medicine Research Ethics Committee of Tongji Hospital of Huazhong University of Science and Technology (No. TJ-IRB20192511), and all stroke patients gave informed consent before enrollment.

Open Access Statement: This is an Open Access article distributed in accordance with the Creative Commons Attribution-NonCommercial-NoDerivs 4.0 International License (CC BY-NC-ND 4.0), which permits the noncommercial replication and distribution of the article with the strict proviso that no changes or edits are made and the original work is properly cited (including links to both the formal publication through the relevant DOI and the license). See: https://creativecommons.org/licenses/by-nc-nd/4.0/.

\section{References}

1. Franco M, Cooper RS, Bilal U, Fuster V. Challenges and opportunities for cardiovascular disease prevention. Am J Med 2011;124:95-102.

2. Fugate JE, Giraldo EA, Rabinstein AA. Thrombolysis for cerebral ischemia. Front Neurol 2010;1:139.

3. Sharma VK, $\mathrm{Ng} \mathrm{KW}$, Venketasubramanian N, et al. Current status of intravenous thrombolysis for acute ischemic stroke in Asia. Int J Stroke 2011;6:523-30.

4. Chen Y, Zhang C, Wang X, et al. Suitability of Thrombolysis for patients with acute ischemic stroke complicated with Trousseau syndrome. Front Neurosci 2020;14:481.

5. Székely EG, Czuriga-Kovacs KR, Bereczky Z, et al. Low factor XIII levels after intravenous thrombolysis predict short-term mortality in ischemic stroke patients. Sci Rep 2018;8:7662.

6. Hou X, Chen H. Proposed antithrombotic strategy for acute ischemic stroke with large-artery atherosclerosis: focus on patients with high-risk transient ischemic attack and mild-to-moderate stroke. Ann Transl Med 2020;8:16.

7. Tanne D, Kasner Se, Demchuk AM, et al. Markers 
of increased risk of intracerebral hemorrhage after intravenous recombinant tissue plasminogen activator therapy for acute ischemic stroke in clinical practice: the Multicenter rt-PA Stroke Survey. Circulation 2002;105:1679-85.

8. Selim M, Fink J, Kumar S, et al. Predictors of hemorrhagic transformation after intravenous recombinant tissue plasminogen activator. Prognostic value of the initial apparent diffusion coefficient and diffusion-weighted lesion volume. Stroke 2002;33:2047-52.

9. Ribo M, Montaner J, Molina C, et al. Admission fibrinolytic profile is associated with symptomatic hemorrhagic transformation in stroke patients treated with tissue plasminogen activator. Stroke 2004;35:2123-7.

10. Johansson L, Jansson JH, Boman K, et al. Tissue plasminogen activator, plasminogen activator inhibitor-1, and tissue plasminogen activator/plasminogen activator inhibitor-1 complex as risk factors for the development of a first stroke. Stroke 2000;31:26-32.

11. Johansson L, Jansson JH, Stegmayr B, et al. Hemostatic factors as risk markers for intracerebral hemorrhage: a prospective incident case-referent study. Stroke 2004;35:826-30.

12. Johansson K, Jansson JH, Johansson L, et al. Factor XII as a risk marker for hemorrhagic stroke: a prospective cohort study. Cerebrovasc Dis Extra 2017;7:84-94.

13. Schwartz ML, Pizzo SV, Hill RL, et al. Human factor XIII from plasma and platelets. Molecular weights, subunit structures, proteolytic activation, and cross-linking of fibrinogen and fibrin. J Biol Chem 1973;248:1395-407.

14. Komáromi I, Bagoly Z, Muszbek L. Factor XIII: novel structural and functional aspects. J Thromb Haemost 2011;9:9-20.

15. Muszbek L, Bereczky Z, Bagoly Z, et al. Factor XIII: a coagulation factor with multiple plasmatic and cellular functions. Physiol Rev 2011;91:931-72.

16. Bagoly Z, Zsuzsa K, Jolan H, et al. Factor XIII, clot structure, thrombosis. Thromb Res 2012;129:382-7.

17. Ariëns RA, Kohler HP, Mansfield MW, et al. Subunit antigen and activity levels of blood coagulation factor $\mathrm{XIII}$ in healthy individuals. Relation to sex, age, smoking, and hypertension. Arterioscler Thromb Vasc Biol 1999; 19:2012-6.

18. Elbaz A, Poirier O, Canaple S, et al. The association between the Val34Leu polymorphism in the factor XIII gene and brain infarction. Blood 2000;95:586-91.

19. Kohler HP, Ariens RA, Catto AJ, et al. Factor XIII A-subunit concentration predicts outcome in stroke subjects and vascular outcome in healthy, middle-aged men. Br J Haematol 2002;118:825-32.

20. Xiao $W$, Chen $W, \mathrm{Hu} H$, et al. The clinical significance of neutrophil gelatinase-associated lipocalin in ischemic stroke patients with acute kidney injury. J Clin Lab Anal 2019;33:e22907.

21. Adams Jr HP, Bendixen BH, Kappelle LJ, et al. Classification of subtype of acute ischemic stroke. Definition for use in a multicenter clinical trial. TOAST. Trial of Org 10172 in Acute Stroke Treatment. Stroke 1993;24:35-41.

22. Brott T, Adams Jr HP, Olinger CP, et al. Measurements of acute cerebral infarction: a clinical examination scale. Stroke 1989;20:864-70.

23. Mittal SH, Goel D. Mortality in ischemic stroke score: a predictive score of mortality for acute ischemic stroke. Brain Circ 2017;3:29-34.

24. Larrue V, von Kummer RR, Muller A, et al. Risk factors of severe hemorrhagic transformation in ischemic stroke patients treated with recombinant tissue plasminogen activator: a secondary analysis of the EuropeanAustralasian Acute Stroke Study (ECASS II). Stroke 2001;32:438-41.

25. Whiteley WN, Emberson J, Lees KR, et al. Risk of intracerebral haemorrhage with alteplase after acute ischaemic stroke: a secondary analysis of an individual patient data meta-analysis. Lancet Neurol 2016;15:925-33.

26. Constant Dit Beaufils P, Preterre C, De Gaalon S, et al. Prognosis and risk factors of asymptomatic intracranial hemorrhage after endovascular treatment of large vessel occlusion stroke: a prospective multicenter cohort study. Eur J Neurol 2021;28:229-37.

27. Menon BK, Saver JL, Prabhakaran S, et al. Risk score for intracranial hemorrhage in patients with acute ischemic stroke treated with intravenous tissue-type plasminogen activator. Stroke 2012;43:2293-9.

28. Saposnik G, Guzik AK, Reeves M, et al. Stroke prognostication using age and NIH stroke scale: SPAN100. Neurology 2013;80:21-8.

29. Van Hooff RJ, Nieboer K, De Smedt A, et al. Validation assessment of risk tools to predict outcome after thrombolytic therapy for acute ischemic stroke. Clin Neurol Neurosurg 2014;125:189-93.

30. Zhou Z, Yin X, Niu Q, et al. Risk factors and a nomogram for predicting intracranial hemorrhage in stroke patients undergoing thrombolysis. Neuropsychiatr Dis Treat 2020;16:1189-97.

31. Mobius C, Blinzler C, Schwab S, et al. Re-evaluation of 
the stroke prognostication using age and NIH Stroke Scale index (SPAN-100 index) in IVT patients - the-SPAN 100(65) index. BMC Neurol 2018;18:129.

32. Lind M, Boman K, Johansson L, et al. D-dimer predicts major bleeding, cardiovascular events and all-cause mortality during warfarin treatment. Clin Biochem 2014;47:570-3.

33. Haapaniemi E, Tatlisumak T. Is D-dimer helpful in evaluating stroke patients? A systematic review. Acta Neurol Scand 2009;119:141-50.

34. Sun X, Berthiller J, Trouillas P, et al. Early fibrinogen degradation coagulopathy: a predictive factor of parenchymal hematomas in cerebral rt-PA thrombolysis. J Neurol Sci 2015;351:109-14.

35. Cocho D, Borrell M, Marti-Fabregas J, et al. Pretreatment hemostatic markers of symptomatic intracerebral hemorrhage in patients treated with tissue plasminogen activator. Stroke 2006;37:996-9.

36. Schroeder V, Ortner E, Mono ML, et al. Coagulation factor FXIII activation peptide and subunit levels in patients with acute ischaemic stroke: a pilot study. Thromb Res 2010;126:e122-7.

37. Stein P, Kaserer A, Sprengel K, et al. Change of

Cite this article as: Zhang L, Zhang C, Luo Y, Tang N. Predictive value of coagulation factor XIII on bleeding risk in ischemic stroke patients treated with intravenous thrombolysis. Ann Palliat Med 2021;10(7):7579-7586. doi: 10.21037/apm-211174 transfusion and treatment paradigm in major trauma patients. Anaesthesia 2017;72:1317-26.

38. Innerhofer P, Fries D, Mittermayr M, et al. Reversal of trauma-induced coagulopathy using first-line coagulation factor concentrates or fresh frozen plasma (RETIC): A single-centre, parallel-group, open-label, randomised trial. Lancet Haematol 2017;4:e258-71.

39. Bagoly Z, Barath B, Orban-Kalmandi R, et al. Incorporation of $\alpha 2$-plasmin inhibitor into fibrin clots and its association with the clinical outcome of acute ischemic stroke patients. Biomolecules 2021;11:347.

40. Koller T, Kinast N, Castellanos AG, et al. Normalization of blood clotting characteristics using prothrombin complex concentrate, fibrinogen and FXIII in an albumin based fluid: experimental studies in thromboelastometry. Scand J Trauma Resusc Emerg Med 2021;29:57.

41. Chuliber FA, Penchasky D, Santoro DM, et al. Acquired factor XIII deficiency in patients under therapeutic plasma exchange: A poorly explored etiology. J Clin Apher 2021;36:59-66.

42. Adam EH, Kreuer S, Zacharowski K, et al. Factor XIII: Pharmacodynamic and pharmacokinetic characteristics. Anaesthesist 2017;66:52-9. 